Published in final edited form as:

Am J Obstet Gynecol. 2018 February ; 218(2): 254.e1-254.e7. doi:10.1016/j.ajog.2017.11.603.

\title{
A validated calculator to estimate risk of cesarean after an induction of labor with an unfavorable cervix
}

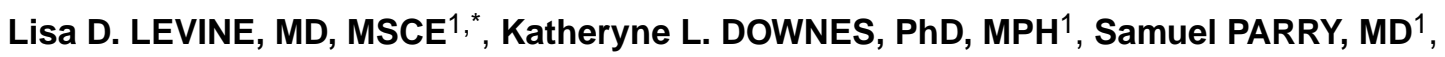 \\ Michal A. ELOVITZ, MD' ${ }^{1}$, Mary D. SAMMEL, ScD ${ }^{2}$, and Sindhu K. SRINIVAS, MD, MSCE ${ }^{1}$ \\ ${ }^{1}$ Maternal and Child Health Research Center, Department of Obstetrics \& Gynecology, University \\ of Pennsylvania Perelman School of Medicine, Philadelphia, PA \\ 2Department of Biostatistics, Epidemiology and Informatics, Center for Clinical Epidemiology and \\ Biostatistics, and Women's Health Clinical Research Center, University of Pennsylvania Perelman \\ School of Medicine, Philadelphia, PA, USA
}

\begin{abstract}
Background-Induction of labor occurs in more than $20 \%$ of pregnancies, which equates to approximately 1 million women undergoing an induction in the United States annually. Regardless of how common inductions are, our ability to predict induction success is limited. Although multiple risk factors for a failed induction have been identified, risk factors alone are not enough to quantify an actual risk of cesarean for an individual woman undergoing a cesarean.
\end{abstract}

Objective-The objective of this study was to derive and validate a prediction model for cesarean after induction with an unfavorable cervix and to create a web-based calculator to assist in patient counseling.

Study Design-Derivation and validation of a prediction model for cesarean delivery after induction was performed as part of a planned secondary analysis of a large randomized trial. A predictive model for cesarean delivery was derived using multivariable logistic regression from a large randomized trial on induction methods $(n=491)$ that took place from 2013-2015 at an academic institution. Full-term ( $>37$ weeks) women carrying a singleton gestation with intact membranes and an unfavorable cervix (Bishop score $\$ 6$ and dilation $\_\mathbf{c m}$ ) undergoing an induction were included in this trial. Both nulliparous and multiparous women were included. Women with a prior cesarean were excluded. Refinement of the prediction model was performed using an observational cohort of women from the same institution who underwent an induction $(\mathrm{n}=364)$ during the trial period. An external validation was performed utilizing a publically

\footnotetext{
"Corresponding author: Lisa D. Levine: Hospital of the University of Pennsylvania, Department of Obstetrics \& Gynecology, 3400 Spruce Street, 2 Silverstein, Philadelphia, PA 19104. Telephone: 516-456-6427, Fax: 215-349-5625, lisa.levine@ uphs.upenn.edu.

Disclosures: L.D.L provided consultant work for Sudler and Hennessey on labor induction which is unrelated to this work. S.K.S. has provided expert testimony in a case on behalf of Pfizer, which is unrelated to this manuscript. She also has a research grant from Bayer through the American Congress of Obstetricians and Gynecologists.

Presentations: The derivation portion of this manuscript was presented as a poster at the Annual Meeting for the Society of Maternal Fetal Medicine in Las Vegas, Nevada in January 2017.

Publisher's Disclaimer: This is a PDF file of an unedited manuscript that has been accepted for publication. As a service to our customers we are providing this early version of the manuscript. The manuscript will undergo copyediting, typesetting, and review of the resulting proof before it is published in its final citable form. Please note that during the production process errors may be discovered which could affect the content, and all legal disclaimers that apply to the journal pertain.
} 
available database (Consortium for Safe Labor) that includes information for $>200,000$ deliveries from 19 hospitals across the United States between 2002-2008. After applying the same inclusion and exclusion criteria utilized in the derivation cohort, a total of 8,466 women remained for analysis. The discriminative power of each model was assessed using a bootstrap, bias-corrected area under the curve (AUC).

Results-The cesarean delivery rates in the derivation and external validation groups were: $27.7 \%(\mathrm{n}=136 / 491)$ and $26.4 \%(\mathrm{n}=2235 / 8466)$. In multivariable modeling, nulliparity, gestation age $\geq 40$ weeks, BMI at delivery, modified Bishop score, and height were significantly associated with cesarean. A nomogram and calculator were created and found to have an area under the curve in the external validation cohort of 0.73 (95\% confidence interval $0.72-0.74$ ).

Conclusion-A nomogram and user-friendly web based calculator which incorporates 5 variables known at the start of induction has been developed and validated. It can be found at: http://www.uphs.upenn.edu/obgyn/labor-induction-calculator/. This calculator can be used to augment patient counseling for women undergoing an induction with an unfavorable cervix.

\section{Keywords}

Bishop score; Calculator; Cesarean; Failed induction; Induction of labor; Unfavorable cervix

\section{Introduction}

In 2012, 23\% of pregnant women (almost 1 million women) underwent an induction of labor. ${ }^{1}$ While it is one of the most common obstetrical procedures, our ability to predict success of induction is limited, despite the fact that approximately one-third of inductions will end in a cesarean delivery. ${ }^{2-5}$

Although multiple risk factors for a failed induction have been identified, ${ }^{2-8}$ risk factors alone are not enough to quantify an actual risk of cesarean for an individual woman undergoing a cesarean. Prediction models for induction success have been limited to nulliparous women and have generally found a favorable starting cervical exam to be the largest driver of success. ${ }^{3-5,9,10}$ Prediction of delivery outcomes for both nulliparous and multiparous women who are starting their induction with an unfavorable cervical exam (Bishop score 56) remains understudied. With the known associated risks of prolonged labor and failed induction, ${ }^{1-13}$ it is clinically useful to be able to accurately predict the likelihood of cesarean after an induction of labor.

Therefore, our objective was to develop and validate a prediction model of cesarean delivery for both nulliparous and multiparous women undergoing an induction of labor with an unfavorable cervix. The goal of this model was to create a calculator that could be used to supplement counseling for women undergoing an induction with an unfavorable cervix.

\section{Materials and Methods}

The current study was a derivation and validation of a prediction model for cesarean delivery after induction of labor. This study was a planned secondary analysis of a large randomized trial (FOR MOMI - Foley or Misoprostol for the Management of Induction) ${ }^{14}$ which 
compared time to delivery among four induction methods (misoprostol alone, cervical Foley alone, misoprostol/cervical Foley concurrently, cervical Foley/oxytocin concurrently). The randomized trial was conducted from May 2013-June 2015 at the Hospital of the University of Pennsylvania. The study was approved by the Institutional Review Board at the University of Pennsylvania. Women with a prior cesarean and contraindication to misoprostol or a vaginal delivery were excluded from the study. ${ }^{14}$ Both nulliparous and multiparous women were included. Full-term ( $>37$ weeks) women carrying a singleton gestation with intact membranes and an unfavorable cervix (Bishop score $\$ 6$ and dilation $\widehat{s} \mathrm{~cm}$ ) undergoing an induction of labor were included. The components of the modified Bishop score were cervical dilation, station, and effacement. ${ }^{15-17}$ Full details regarding the induction and labor protocols utilized for the trial can be found in the original paper. ${ }^{14}$

The primary outcome for the prediction model was cesarean delivery for any indication. The proposed guidelines for model building, refinement and validation for prediction models were utilized. ${ }^{18}$ First, a model was derived using data from a large randomized trial (FOR MOMI, ${ }^{14} \mathrm{n}=491$; derivation dataset). This dataset was chosen for derivation since it was a contemporary dataset (performed from 2013-2015), had an extensive amount of detailed demographic and clinical data available for evaluation, and was collected prospectively from a randomized trial. Prediction models are created by identifying a set of risk factors/ variables that are predictors of the outcome based on multivariable logistic regression modeling. Risk factor selection used stepwise methods considering all variables with at least $5 \%$ prevalence and an association with the outcome (cesarean) of $\mathrm{P}<0.20$ from bivariate tests. Continuous variables were assessed for linearity prior to being entered into models. The following variables were considered for predictive modeling: maternal age, maternal height, maternal weight change over pregnancy, maternal weight change rate, BMI at first prenatal visit, BMI at last prenatal visit, change in BMI over pregnancy, BMI change rate, race, parity, gestational age in weeks at time of induction, postdate gestational age, indication for induction, pre-gestational diabetes, chronic hypertension, presence of oligohydramnios, cervical dilation, station, Bishop score at start of induction, method of induction. Body mass index (BMI) at delivery was defined as the BMI at the time of delivery or at the most recent prenatal visit if delivery BMI was unavailable (for $98 \%$ of women, this was within 1.5 weeks of delivery). Neonatal sex and post-natal weight were also related to delivery outcome, but since they are not always routinely established prior to the start of the induction, they were not included in our models. The final multivariable model included all variables with a $\mathrm{P}<0.05$. Interaction terms between parity and other selected variables were also evaluated. Associations are presented as adjusted odds ratios (ORs) and 95\% confidence intervals (CIs) from the final model. Descriptive statistics for continuous variables are presented as mean and standard deviation or median and interquartile range, where appropriate. Categorical variables are presented as frequencies and percentages. Statistical analyses were performed with STATA version 14 (STATA Corporation, College Station, TX).

Next, the performance of this model was evaluated using an observational cohort of women from the same institution who underwent an induction during the FOR MOMI trial period $(n=364$; internal refinement dataset). Internal refinement of the estimates within a different dataset at the same institution helps improve the precision of those estimates and to ensure 
the original estimates do not over-estimate or under-estimate the results. These women met all inclusion criteria for the FOR MOMI trial but declined enrollment into the trial and consented to data abstracted as part of an observational control group. As compared to the FOR MOMI trial that utilized protocols for the management of induction and labor progress, 14 the management of labor in this observational group was at the discretion of the provider with no specific protocols or recommendations for labor management or cesarean. The same inclusion and exclusion criteria utilized in the derivation cohort were also applied to this internal validation cohort. We applied the initial model to this cohort, assessed performance of the model and then re-estimated and refined the associations between risk factors and cesarean delivery.

Thirdly, an external validation of the refined prediction model was then performed using the Consortium for Safe Labor database (validation dataset) ${ }^{19}$ to ensure the model has the same predictive ability as in the derivation cohort. The Consortium for Safe Labor database is a publicly available NIH database that includes labor and delivery information collected from the electronic medical record for over 200,000 deliveries from 19 hospitals across the United States between 2002-2008. 3,20,21 The same inclusion and exclusion in the derivation cohort was applied to the validation dataset. After applying these criteria, a total of 8,466 women remained for analysis. The discriminative power of each model was assessed using area under the receiver operating characteristic (AUC) curve. This metric summarizes the sensitivity and specificity of the predicted model for every possible choice of cut-off, and is a useful metric to summarize how a model can discriminate among cesarean and vaginal delivery outcomes. We present bootstrap, bias-corrected area under the curve (AUC) for each model. ${ }^{18}$ Finally, a nomogram based on the final model was created. A nomogram is derived from estimating the predicted probability of cesarean using logistic regression coefficients from the logistic model. 22,23 This was done using the STATA "nomolog" package and a corresponding, user-friendly on-line calculator was created.

\section{Results}

There were 491 women included in the derivation cohort with a cesarean delivery rate of $27.7 \%(n=136 / 491)$. There were 364 women in the internal refinement cohort with a cesarean rate of $32.7 \%(n=119 / 364)$ and 8,466 women in the external validation samples with a cesarean rate of $26.4 \%(\mathrm{n}=2235 / 8466)$. There were no differences in cesarean rate between the derivation group and external validation group $(\mathrm{p}=0.53)$. Characteristics of the derivation cohort by mode of delivery are presented in Table 1. Within the derivation group, indications for cesarean were as follows: $51.4 \%$ for failed induction or arrest of labor, $10.3 \%$ for arrest of descent, and $34.6 \%$ for fetal indication. In bivariate analysis, the following variables had a $\mathrm{P}<0.20$ and were considered in the development of the prediction model: maternal height, weight gain during pregnancy, BMI at delivery, race, parity, gestational age at induction, indication for induction, pre-existing diabetes, chronic hypertension, cervical dilation at start of induction, and the modified Bishop scores at start of induction.

Table 2 shows the estimates for the derivation cohort and internal refinement cohort. BMI and height were both found to have non-linear relationships with the outcome and were ultimately converted to ordinal variables. In the multivariable modeling, nulliparity, 
gestational age at induction $\geq 40$ weeks, BMI category at delivery, the modified Bishop score at induction, and height category were the five variables that remained significantly associated with cesarean. Cervical dilation at induction was not found to be associated with cesarean delivery (OR 0.95 [0.57-1.59]). Method of induction was also not associated with cesarean delivery. Because of this, when induction method was added into the model, there was no change in estimates and therefore was not forced into the model. Finally, there was no evidence of interaction between parity and height, BMI, gestational age $\geq 40$ weeks, or Bishop score ( $p>0.10$ for all interactions) and therefore results were not stratified by parity.

A nomogram based on the final, refined estimates with these variables was constructed and is shown in Figure 1. The final model was also used to create a user-friendly web based calculator that gives the percentage likelihood of cesarean delivery (http:// www.uphs.upenn.edu/obgyn/labor-induction-calculator/). External validation of this scoring system and model was performed using the Consortium for Safe Labor dataset $(\mathrm{n}=8,466$, cesarean incidence $26.4 \%$ ). The final area AUC was 0.73 (95\% CI: 0.72-0.74). Figure 2 displays the predicted probability of cesarean based on the calculator (x-axis) compared to the actual cesarean rate (y-axis).

\section{Comment}

\section{Main Findings}

We found maternal BMI at delivery, height, parity, gestational age $\geq 40$ weeks at induction, and modified Bishop score to be independent risk factors for cesarean delivery among women undergoing an induction of labor with an unfavorable cervix. Using these factors, we developed and externally validated a model and created an on-line tool that can be utilized to calculate the likelihood of cesarean for women undergoing induction with an unfavorable cervix.

\section{Clinical Implications}

Risk calculators like this one have been incorporated into the Obstetrical community and, importantly, should not be used in isolation to make clinical decisions. ${ }^{24,25}$ Instead, information obtained from calculators such as the NICHD calculator for extreme prematurity outcomes ${ }^{24}$ or the VBAC calculator ${ }^{23,25}$ is used in the overall context of clinical information to help with patient counseling and expectations. Similar to these other calculators, the induction of labor calculator also should not be used in isolation when making clinical decisions. The results of the calculator should be used to augment counseling of patients regarding the risks of an induction and to set expectations for a patient regarding these risks. The results may also be helpful in clinical scenarios when expectant management is an option. It is important to note that the calculator reports a percentage likelihood of cesarean and there is no discrete cut-off of risk above which a cesarean is indicated. Results from the calculator should not be used to forego an induction when it is medically indicated, to lower the threshold for elective primary cesarean delivery, or to lower the threshold for cesarean delivery in labor in the absence of other clinical information. 


\section{Strengths and Weaknesses}

The characteristics that we identified as risk factors for cesarean delivery have been previously reported in the literature ${ }^{3-8}$ however, they have not been compiled in a risk model that estimates the percent likelihood of cesarean based on those factors. Our study differs from the current literature by including both nulliparous and multiparous women as well as including only women whose starting cervical exam is unfavorable (cervical dilation $\widehat{2} \mathrm{~cm}$ and Bishop score was $\mathbf{6}$ ). While Bishop score was noted to be predictive, cervical dilation alone was not. This is likely due to the fact that all women were starting out unfavorable with minimal dilation. Including only women with an unfavorable cervix is an important difference and strength of our study since most of the prior work identified a "favorable" cervix as being a large driver of success ${ }^{3-5,9,10}$ thereby limiting our predictive ability for women with an unfavorable starting cervical exam. Importantly, in addition to deriving a predictive model for cesarean, we also both internally and externally validated the model. This is critical when interpreting the reliability of a model that may be used for clinical care and ensuring its reproducibility in a more generalized population. Another strength of our study is that the derivation model was created within a population of contemporary data from a randomized trial, limiting the biases obtained from retrospective data. Since the randomized trial had labor protocols utilized, it was also important to validate the study in a population where management was at the discretion of the provider to ensure these results were generalizable.

This predictive model is limited to the women who met inclusion criteria for our study and may not be generalizable to women who do not fit these criteria. For example, it is not recommended this calculator be used in women with a prior cesarean, women with ruptured membranes, or women with a favorable cervix as the predictive ability in those populations is not known. Although the model was derived in an academic population, the external validation in the Consortium for Safe Labor database increases generalizability to both community and non-academic hospitals. A limitation of this model is the inability to reliably predict cesarean with $100 \%$ accuracy. However, given the fact there will always be confounding by indication and provider clinical decision making that goes into choosing to perform a cesarean delivery, achieving $100 \%$ accuracy in a prediction model like this would not be realistic.

\section{Conclusion/Direction of Future Research}

An example of how the on-line calculator works is shown in Figure 3 and described here. When using the calculator for a nulliparous woman at 39 weeks who is 65 inches tall with a BMI of $29 \mathrm{~kg} / \mathrm{m}^{2}$ and a starting modified Bishop score at induction of 4 , her probability of cesarean delivery is $19.3 \%$. If a multiparous woman at 41 weeks who is 63 inches tall with a BMI of $40 \mathrm{~kg} / \mathrm{m}^{2}$ is undergoing an induction with a starting modified Bishop score of 3 , her probability of cesarean delivery is $31.7 \%$.

With approximately one million women undergoing an induction in the United States annually, it is critical to be able to appropriately counsel women about the risks and benefits of an induction. This web based calculator can be used as a tool to help augment patient counseling and guide patient expectations in both academic and community practices. It is a 
novel tool that can offer a personalized medicine and evidence based approach to counseling women regarding the risk of cesarean when undergoing an induction with an unfavorable cervix. Future studies should focus on the maternal and neonatal morbidity associated with a high predicted cesarean risk in order to further aid in patient counseling and decision making.

\section{Acknowledgments}

Sources of funding: This study was funded in part by a career development award in Women's Reproductive Health Research: K12-HD001265-15 and the Maternal and Child Health Research fund from the University of Pennsylvania.

\section{References}

1. Center for Disease Control and Prevention. [Accessed March 1, 2016] Recent declines in induction of labor. http://www.cdc.gov/nchs/data/databriefs/db155.htm

2. Rouse DJ, Weiner SJ, Bloom SL, et al. Failed labor induction: toward an objective diagnosis. Obstet Gynecol. 2011; 117(2 Pt 1):267-272. [PubMed: 21252738]

3. Vahratian A, Zhang J, Troendle JF, Sciscione AC, Hoffman MK. Labor progression and risk of cesarean delivery in electively induced nulliparas. Obstet Gynecol. 2005; 105(4):698-704. [PubMed: 15802393]

4. Grobman WA. Predictors of induction success. Semin Perinatol. 2012; 36(5):344-347. [PubMed: 23009966]

5. Tolcher MC, Holbert MR, Weaver AL, et al. Predicting Cesarean Delivery After Induction of Labor Among Nulliparous Women at Term. Obstet Gynecol. 2015; 126(5):1059-1068. [PubMed: 26444107]

6. Beckwith L, Magner K, Kritzer S, Warshak CR. Prostaglandin versus mechanical dilation and the effect of maternal obesity on failure to achieve active labor: a cohort study. J Matern Fetal Neonatal Med. 2017; 30(13):1621-1626. [PubMed: 27560557]

7. Ennen CS, Bofill JA, Magann EF, Bass JD, Chauhan SP, Morrison JC. Risk factors for cesarean delivery in preterm, term and post-term patients undergoing induction of labor with an unfavorable cervix. Gynecol Obstet Invest. 2009; 67(2):113-117. [PubMed: 18971583]

8. Crane JM. Factors predicting labor induction success: a critical analysis. Clin Obstet Gynecol. 2006; 49(3):573-584. [PubMed: 16885664]

9. Vrouenraets FP, Roumen FJ, Dehing CJ, van den Akker ES, Aarts MJ, Scheve EJ. Bishop score and risk of cesarean delivery after induction of labor in nulliparous women. Obstet Gynecol. 2005; 105(4):690-697. [PubMed: 15802392]

10. Yeast JD, Jones A, Poskin M. Induction of labor and the relationship to cesarean delivery: A review of 7001 consecutive inductions. Am J Obstet Gynecol. 1999; 180(3 Pt 1):628-633. [PubMed: 10076139]

11. Spong CY, Berghella V, Wenstrom KD, Mercer BM, Saade GR. Preventing the first cesarean delivery: summary of a joint Eunice Kennedy Shriver National Institute of Child Health and Human Development, Society for Maternal-Fetal Medicine, and American College of Obstetricians and Gynecologists Workshop. Obstet Gynecol. 2012; 120(5):1181-1193. [PubMed: 23090537]

12. Cheng YW, Shaffer BL, Bryant AS, Caughey AB. Length of the first stage of labor and associated perinatal outcomes in nulliparous women. Obstet Gynecol. 2010; 116(5):1127-1135. [PubMed: 20966698]

13. Maghoma J, Buchmann EJ. Maternal and fetal risks associated with prolonged latent phase of labour. J Obstet Gynaecol. 2002; 22(1):16-19. [PubMed: 12521720]

14. Levine LD, Downes KL, Elovitz MA, Parry S, Sammel MD, Srinivas SK. Mechanical and Pharmacologic Methods of Labor Induction: A Randomized Controlled Trial. Obstet Gynecol. 2016; 128(6):1357-1364. [PubMed: 27824758] 
15. Lange AP, Secher NJ, Westergaard JG, Skovgard I. Prelabor evaluation of inducibility. Obstet Gynecol. 1982; 60(2):137-147. [PubMed: 7155472]

16. Wing DA, Brown R, Plante LA, Miller H, Rugarn O, Powers BL. Misoprostol vaginal insert and time to vaginal delivery: a randomized controlled trial. Obstet Gynecol. 2013; $122(2 \mathrm{Pt} 1): 201-$ 209. [PubMed: 23857539]

17. Osmundson S, Ou-Yang RJ, Grobman WA. Elective induction compared with expectant management in nulliparous women with an unfavorable cervix. Obstet Gynecol. 2011; 117(3): 583-587. [PubMed: 21343761]

18. Moons KG, Altman DG, Reitsma JB, et al. Transparent Reporting of a multivariable prediction model for Individual Prognosis or Diagnosis (TRIPOD): explanation and elaboration. Ann Intern Med. 2015; 162(1):M14-0698.

19. NICHD. [Accessed April 4, 2017] Epidemiology Branch-Perinatal Epidemiology. Consortium on Safe Labor. https://www.nichd.nih.gov/about/org/diphr/eb/research/Pages/safe-labor.aspx

20. Zhang J, Landy HJ, Branch DW, et al. Contemporary patterns of spontaneous labor with normal neonatal outcomes. Obstet Gynecol. 2010; 116(6):1281-1287. [PubMed: 21099592]

21. Laughon SK, Zhang J, Grewal J, Sundaram R, Beaver J, Reddy UM. Induction of labor in a contemporary obstetric cohort. Am J Obstet Gynecol. 2012; 206(6):23.

22. Iasonos A, Schrag D, Raj GV, Panageas KS. How to build and interpret a nomogram for cancer prognosis. J Clin Oncol. 2008; 26(8):1364-1370. [PubMed: 18323559]

23. Grobman WA, Lai Y, Landon MB, et al. Development of a nomogram for prediction of vaginal birth after cesarean delivery. Obstet Gynecol. 2007; 109(4):806-812. [PubMed: 17400840]

24. NICHD NRN. [Accessed August 28, 2017] Extremely Preterm Birth Outcome Data. https:// www.nichd.nih.gov/about/org/der/branches/ppb/programs/epbo/Pages/epbo_case.aspx

25. [Accessed August 28, 2017] MFMU VBAC Calculator. https://mfmunetwork.bsc.gwu.edu/ PublicBSC/MFMU/VGBirthCalc/vagbirth.html 


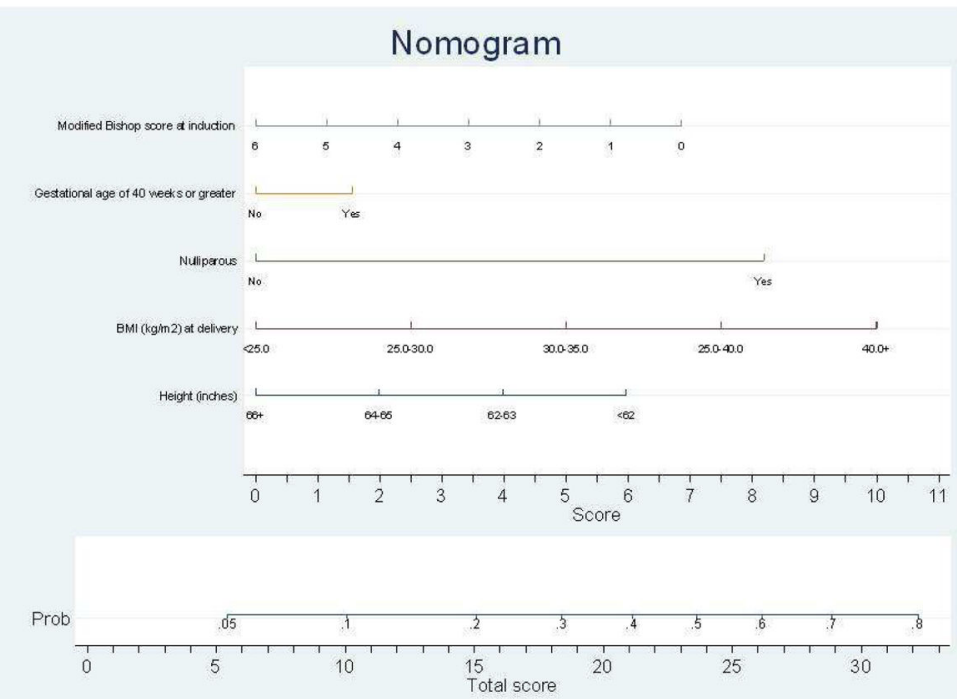

Figure 1.

Nomogram for predicting probability of cesarean delivery

BMI: Body Mass Index

This Figure is a Nomogram that can be used to calculate the predicted probability of cesarean delivery based on the validated model. 


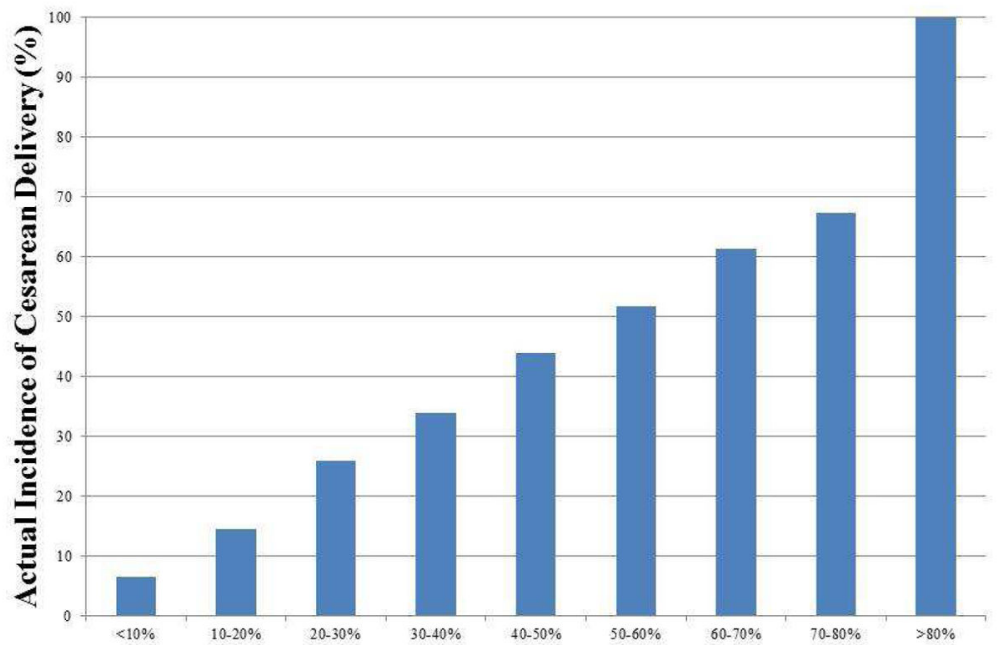

Predicted Probability of Cesarean Delivery

Figure 2.

Cesarean delivery incidence by predicted probability category

This Figure displays predicted probability of cesarean based on the calculator (x-axis) and the actual cesarean rate on (y-axis) 


\begin{tabular}{|c|c|}
\hline \multicolumn{2}{|c|}{ Labor Induction Calculator } \\
\hline 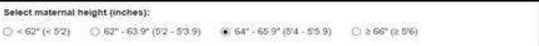 & 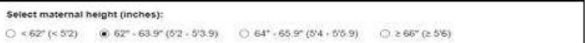 \\
\hline 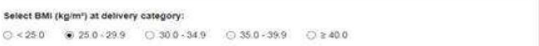 & 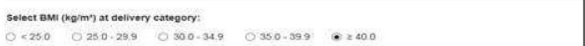 \\
\hline 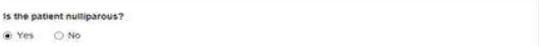 & 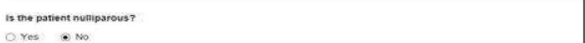 \\
\hline 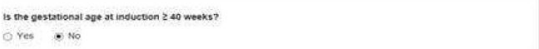 & 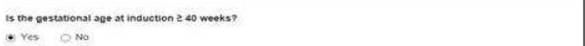 \\
\hline 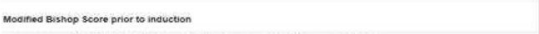 & 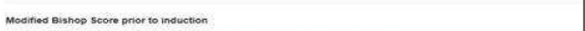 \\
\hline 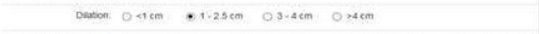 & 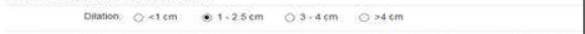 \\
\hline 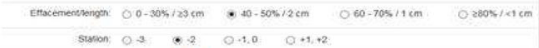 & 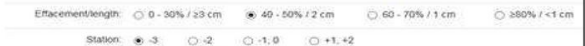 \\
\hline 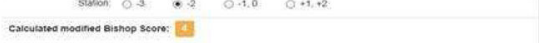 & 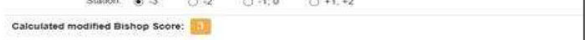 \\
\hline 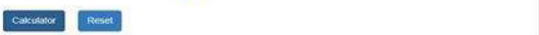 & 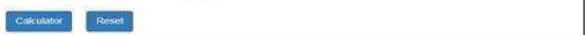 \\
\hline Probability of cesarean dell & Probability of cesarean delivery: उ1:73 \\
\hline
\end{tabular}

Figure 3.

Screenshot of Web Based Labor Induction Calculator

This is a screenshot of two examples of how the web based calculator works. 
Table 1

Characteristics of women from the derivation group by mode of delivery

\begin{tabular}{|c|c|c|c|}
\hline Characteristic & Vaginal Delivery n (\%) & Cesarean Delivery n (\%) & Pvalue ${ }^{a}$ \\
\hline Maternal Age, years $b$ & $28(22-32)$ & $25(22-32)$ & 0.26 \\
\hline \multicolumn{4}{|l|}{ Height, inches } \\
\hline$<62^{\prime \prime}$ & $44(12.4)$ & $23(16.9)$ & \multirow{4}{*}{0.05} \\
\hline $62-63.9^{\prime \prime}$ & $68(19.2)$ & $38(27.9)$ & \\
\hline $64-65.9^{\prime \prime}$ & $108(30.5)$ & $31(22.8)$ & \\
\hline $266^{\prime \prime}$ & $134(37.8)$ & $44(32.3)$ & \\
\hline \multicolumn{4}{|l|}{ BMI at delivery, $\mathrm{kg} / \mathrm{m}^{2}$} \\
\hline$<25.0$ (Normal weight) & $23(6.9)$ & $6(4.7)$ & \multirow{5}{*}{0.005} \\
\hline 25.0-29.9 (Overweight) & $97(29.3)$ & $26(20.5)$ & \\
\hline 30.0-34.9 (Obese Class 1) & $87(26.3)$ & $23(18.1)$ & \\
\hline 35.0-39.9 (Obese Class 2) & $63(19.0)$ & $42(33.1)$ & \\
\hline$\geq 40.0$ (Obese Class 3 ) & $61(18.4)$ & $30(23.6)$ & \\
\hline \multicolumn{4}{|l|}{ Race } \\
\hline White & $50(14.1)$ & $26(19.1)$ & \multirow{3}{*}{0.18} \\
\hline Black & $283(79.7)$ & $98(72.1)$ & \\
\hline Other & $22(6.2)$ & $12(8.8)$ & \\
\hline Nulliparity & $174(49.0)$ & $116(85.3)$ & $<0.001$ \\
\hline \multicolumn{4}{|l|}{ Gestational age at delivery, weeks } \\
\hline $37 w 0 d-37 w 6 d$ & $83(23.4)$ & $22(16.2)$ & \multirow{5}{*}{$<0.001$} \\
\hline $38 w 0 d-38 w 6 d$ & $64(18.0)$ & $13(9.6)$ & \\
\hline $39 w 0 d-39 w 6 d$ & $98(27.6)$ & $24(17.6)$ & \\
\hline 40w0d-40w6d & $71(20)$ & $34(25)$ & \\
\hline $241 w$ & $39(11)$ & $43(31.6)$ & \\
\hline \multicolumn{4}{|l|}{ Indication for induction } \\
\hline Postdate & $26(7.3)$ & $38(27.9)$ & \multirow{4}{*}{$<0.001$} \\
\hline Maternal $^{c}$ & $114(32.1)$ & $34(25)$ & \\
\hline Fetal $^{d}$ & $170(47.9)$ & $55(40.4)$ & \\
\hline Elective & 45 (12.7) & $9(6.6)$ & \\
\hline Pre-existing or Gestational diabetes & $28(7.9)$ & $16(11.8)$ & 0.22 \\
\hline Chronic Hypertension & $34(9.6)$ & $6(4.4)$ & 0.07 \\
\hline Hypertensive disease of pregnancy & $111(31.3)$ & $53(39.0)$ & 0.11 \\
\hline Cervical dilation at induction, $\mathrm{cm}^{b}$ & $1(1-2)$ & $1(0.5-1.5)$ & $<0.001$ \\
\hline Effacement at induction, $\mathrm{cm}^{b}$ & $2(2-3)$ & $2(2-3)$ & 0.45 \\
\hline Station at induction, $\mathrm{cm}^{b}$ & $-3(-3$ to -3$)$ & $-3(-3$ to -3$)$ & 0.44 \\
\hline Modified Bishop score at induction $b$ & $3(2-4)$ & $2(2-3)$ & 0.006 \\
\hline
\end{tabular}




\begin{tabular}{|l|c|c|c|}
\hline Characteristic & Vaginal Delivery n (\%) & Cesarean Delivery n (\%) & \multirow{2}{*}{ P value } \\
\cline { 1 - 3 } Method of Induction/Cervical Ripening & $96(27.0)$ & $33(24.3)$ & \\
\cline { 1 - 3 } Misoprostol alone & $81(22.8)$ & $31(22.8)$ & \multirow{2}{*}{0.85} \\
\cline { 1 - 3 } Misoprostol + cervical Foley & $89(25.1)$ & $33(24.3)$ & \\
\cline { 1 - 3 } Cervical Foley alone & $81(22.8)$ & $34(25.0)$ & \\
\cline { 1 - 3 } Cervical Foley + oxytocin & $8(2.2)$ & $5(3.7)$ & \\
\cline { 1 - 3 } & &
\end{tabular}

Abbreviations: IQR, interquartile; BMI, body mass index; GHTN, gestational hypertension; range; PEC, preeclampsia

Data are presented as $\mathrm{n}(\%)$ unless otherwise indicated

${ }^{a}$ Two sided P-value based on $\chi^{2}$ or Fisher's exact for categorical variables and Wilcoxon rank-sum for all continuous variables;

${ }^{b}$ Median (inter-quartile range);

${ }^{c}$ Examples of maternal indications include: chronic hypertension, gestational diabetes, history of venous thromboembolism, pre-gestational diabetes, pregnancy related hypertension, renal disease, systemic lupus erythematous;

$d_{\text {Examples of fetal indications include: abnormal fetal testing, growth restriction, oligohydramnios }}$ 


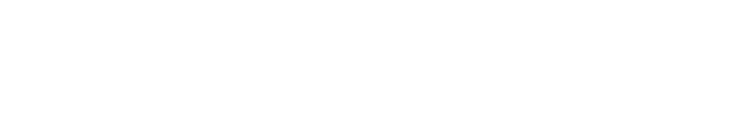

